\title{
CRYSTALLOGRAPHY OF BOUNDARIES AND INTERFACES
}

\author{
W. NEUMANN \\ Max-Planck-Institut für Mikrostrukturphysik \\ Weinberg 2, 06120 Halle/Saale, Germany
}

The main crystallographic concepts of characterizing interface structures are treated and reviewed. It will be demonstrated in which way the approaches of interface crystallography can be used to analyse interface structures experimentally observed.

PACS numbers: $68.55 . \mathrm{Jk}, 68.35 . \mathrm{Bs}$

\section{Introduction}

Internal interfaces in solids influence and/or even control essential properties of materials. In order to have a thorough understanding of structure-property correlations, systematical investigations of the atomic structure of interfaces and boundaries are necessary. The atomic structure of interfaces and boundaries can be determined by high-resolution electron microscopy (HREM). The different techniques of analytical electron microscopy (energy-dispersive X-ray spectroscopy EDX, electron energy loss spectroscopy - EELS, and convergent beam electron diffraction - CBED) provide the required information on the chemical composition of the interface regions. In order to determine the equilibrium structure and the energy of an interface, atomistic simulations of the interface structure have to be carried out. The relaxed interface structure may be simulated applying molecular dynamical (MD) calculations and the methods of static energy minimization (MS). For all interface calculations a suitable geometrical model has to be generated (construction of the computational cell). Then, the interatomic interaction process as well as the relaxation process have to be taken into consideration.

In the following various crystallographic concepts of characterizing interface structures are treated. In general, the determination of the symmetry of the two adjacent crystals and of that of the interfaces between them is very important both for the analysis and the prediction of possible interface structures.

A useful classification scheme for crystalline interfaces is to distinguish between homophase- and heterophase boundaries, sometimes also denoted as grain boundaries and interphase boundaries, respectively.

Homophase boundaries are interfaces between two crystals with the same crystal structure and the same chemical composition. Grain boundaries and other 
species of theirs like twins, domain boundaries, stacking faults, antiphase boundaries, and inversion boundaries are homophase boundaries.

Heterophase boundaries are interfaces between two crystals where either the lattices and/or the bases are different. Examples of heterophase boundaries are, for instance, interfaces of thin films, boundaries in the various types of composites, multi-layers, precipitates, polytypes, and polysomes.

The atomic structure of interfaces is very often associated with heterogeneities caused by the interaction between the adjacent crystals as a function of crystal structure, lattice parameters and bonding. The real atomic interface structure may contain various structural and chemical defects as, e.g., facets, steps, line defects, cracks, voids, atomic disorder, segregation, precipitates, etc.

Therefore, it is useful to subclassify interfaces into sharp and diffuse ones with respect to their atomic structure [1]. The width of a sharp interface is of the order of a nearest-neighbour separation whereas the width of a diffuse one is large compared to a nearest-neighbour separation. Another point of classifying interfaces concerns the matching of the lattices along the interfaces. Thus, interfaces may be divided into coherent, semi-coherent and incoherent interfaces. Concerning the periodicity in the interface plane one can divide the interfaces of the semi-coherent type into commensurate and incommensurate ones. A commensurate interface is characterized by a one- or two-dimensional periodicity in the interface plane. An incommensurate interface is given if the translation vectors of the adjoining parts of the bicrystal in each direction within the interface are irrational multiples of each other.

The complexity of a common terminology to characterize the different structural aspects of crystalline interfaces will not be treated here (for a detailed discussion, see e.g. $[1,2])$.

\section{Symmetry of crystals - some basic facts on crystallographic groups}

In the following, some basic facts of the symmetry concept are briefly summarized, which are necessary for the application to describe the symmetry of interfaces and boundaries (for a deeper insight, see e.g. [3-7]).

Crystals are finite real objects being mesoscopically homogeneous and microscopically periodic. Classical crystallography describes the symmetry of the ideal crystals neglecting the influence of crystal surfaces as well as any structural imperfections. The theory assumes a perfect infinite crystal, the structure of which at the level of atomic arrangements is three-dimensional periodic. The symmetry groups of the crystal structures are the space groups. There is an infinite number of them. According to their symmetry all the crystal structures can be classified in one of the 230 space group types. The elements of the groups are the possible crystallographic symmetry operations (rotation, including the identity operation $I$, reflection, inversion, rotoinversion, translation, screw rotation, glide reflection), which can be represented by matrix-column pairs $(W, w)$ or by $(4 \times 4)$ augmented matrices $W$ for a chosen coordinate system. Matrix part $W$ (rotation part) only depends on the choice of the basis, whereas column part $w$ (translational part) depends on the choice of the basis and the origin $\left(w=w_{\mathrm{g}}+w_{\mathrm{l}} ; w_{\mathrm{g}}-\right.$ translation part, $w_{1}$ - location part). In general, the mapping of any given symmetry 
operation in a space group referring to a coordinate system $\left(O, a_{1}, a_{2}, a_{3}\right)$ can be described by the following:

$$
\begin{aligned}
& \left(\begin{array}{c}
\tilde{x}_{1} \\
\tilde{x}_{2} \\
\tilde{x}_{3}
\end{array}\right)=\left(\begin{array}{lll}
W_{11} & W_{12} & W_{13} \\
W_{21} & W_{22} & W_{23} \\
W_{31} & W_{32} & W_{33}
\end{array}\right)\left(\begin{array}{l}
x_{1} \\
x_{2} \\
x_{3}
\end{array}\right)+\left(\begin{array}{l}
w_{1} \\
w_{2} \\
w_{3}
\end{array}\right), \\
& \tilde{x}=W x+w=(W, w) x .
\end{aligned}
$$

Here, $x_{i}, \tilde{x}_{i}$ are the coordinates of an atom before and after the symmetry operation. The general form of the augmented matrix is given by

$$
W=\left(\begin{array}{ccc|c} 
& W & & w \\
& & & \\
\hline 0 & 0 & 0 & 1
\end{array}\right) .
$$

Thus, a space group is a set of all crystallographic symmetry operations (isometries) mapping the three-dimensional periodic object onto itself. In order to illustrate the architecture of a space group the general form of a coset decomposition of the space group $G$ relative to the normal subgroup $T$ of all translations is given by

$$
\begin{array}{cccccc}
(I, 0) & \left(W_{2}, w_{2}\right) & \ldots & \left(W_{m}, w_{m}\right) & \ldots & \left(W_{i}, w_{i}\right) \\
\left(I, t_{1}\right) & \left(W_{2}, w_{2}+t_{1}\right) & \ldots & \left(W_{m}, w_{m}+t_{1}\right) & \ldots & \left(W_{i}, w_{i}+t_{1}\right) \\
\left(I, t_{2}\right) & \left(W_{2}, w_{2}+t_{2}\right) & \ldots & \left(W_{m}, w_{m}+t_{2}\right) & \ldots & \left(W_{i}, w_{i}+t_{2}\right) \\
\vdots & \vdots & & \vdots & & \vdots \\
\left(I, t_{j}\right) & \left(W_{2}, w_{2}+t_{j}\right) & \ldots & \left(W_{m}, w_{m}+t_{j}\right) & \ldots & \left(W_{i}, w_{i}+t_{j}\right)
\end{array}
$$

Translation subgroup $T$ is of infinite order, Abelian, and is represented by the elements $\left(I, t_{j}\right)$ forming the first column. The number of cosets of $T$ in $G$ is calied the index $i$ of $T$ in $G$ and denoted by $i=[G: T]$. The index $i$ is equal to the order of the point group $i$, i.e. ranging from a minimum of 1 to a maximum of 48. The matrices $I, W_{2}, \ldots W_{m}$ represent the point group $P$ belonging to $G$. There are 32 point groups in three-dimensional space, determining the symmetry of the ideal external crystal shape, of the diffraction patterns as well as of the physical properties (tensor properties) of the crystals. As can be seen from the coset decomposition, space groups are connected with a given point group, a Bravais lattice, and the action of the point group on the lattice.

Group-subgroup relations $G-H$ of space groups are important, for instance, for the description of homophase boundaries generated during phase transitions which involve a change in the space group symmetry.

Translationengleiche subgroups ( $t$-subgroups) are given if the set of all translations is retained $(T(H)=T(G))$ and the order of the point group symmetry is reduced, i.e. by eliminating all symmetry operations of some kind $(P(H)<P(G))$.

Klassengleiche subgroups ( $k$-subgroups) are given if the point group symmetry remains unchanged $(P(H)=P(G))$. The symmetry dilution occurs by loss of translational symmetry either by the loss of a centering translation and/or by a 
change in size of the unit cell $(T(H)<T(G))$. A $k$-subgroup is called isomorphic if it belongs not only to the same crystal class but even to the same space group type as $G$ or to its enantiomorphic.

The symmetry reduction may, of course, also occur by a combination of dilution of translational and point group symmetry elements. According to the theorem of Hermann that type of subgroups $(P(H)<P(G), T(H)<T(G))$ cannot be a maximal subgroup [8]. A maximal subgroup of $H$ of $G$ is either translationengleich or klassengleich. Thus, there must exist a space group $Z$ for which yields

$$
H \stackrel{k}{<} Z \stackrel{t}{<} G \text {. }
$$

Group-subgroup relationships are useful for determining both the boundary type and the possible number of domains (or variants) generated during a phase transition (for a detailed group-theoretical treatment, see [9, 10]).

Twin boundaries arise if the low symmetry phase is a $t$-subgroup of the high symmetry phase. Merohedral twins will be formed if the two phases are of the same Bravais lattice. Electron diffraction patterns of merohedral twins do not show a spot splitting. It should be mentioned that inversion domains are merohedral twins if the domains are related by a centre of symmetry. Pseudo-merohedral twins (e.g. ferroelastic twins) are formed if the low symmetry phase has got another Bravais lattice than the high symmetry phase has. Because of a small mismatch between the two lattices the electron diffraction patterns of pseudo-merohedral twins show a spot splitting.

Antiphase domains arise if the low symmetry phase is a $k$-subgroup of the high symmetry one.

As it was previously mentioned the degree of symmetry reduction is given by the index $i$. The number of possible domains or variants is determined by the $t$-index and $k$-index, respectively.

The $t$-index is given by

$$
t=G_{\mathrm{p}} / H_{\mathrm{p}}
$$

( $G_{\mathrm{p}}, H_{\mathrm{p}}$ - order of the point groups). The $k$-index is given by

$$
k=\left(V_{\mathrm{h}} \cdot T_{\mathrm{g}}\right) /\left(T_{\mathrm{h}} \cdot V_{\mathrm{g}}\right)
$$

$\left(V_{\mathrm{h}}, V_{\mathrm{g}}\right.$ - volumes of the cells, $\mathrm{T}_{\mathrm{h}}, T_{\mathrm{g}}$ - numbers of lattice points). In general, a phase transition, where the low symmetry phase is a general subgroup, results in a complicated domain pattern.

In order to describe the symmetry of interfaces and boundaries it is useful to apply the generalized symmetry concept of antisymmetry operations $W^{\prime}$ leading to the antisymmetry groups (Shubnikov-Heesch groups) also known as black-white groups $[11,12]$. Referring to the point group symmetry the antisymmetry concept can be described as follows. In addition to the identity operation $1(I)$ there is the antiidentity operation $1^{\prime}\left(I^{\prime}\right)$. The antiidentity means that the two objects differ only in colour $\left(1^{\prime} \circ 1^{\prime}=1\right)$. Antisymmetry operations may be written as the product of an ordinary symmetry operation and the antiidentity operation

$$
W_{i^{\prime}}=W_{i} \circ 1^{\prime} \text {. }
$$

The multiplication of ordinary symmetry operations and antisymmetry operations yields

$$
W_{i} \circ W_{j}=W_{k} \quad \rightarrow \text { symmetry operation, }
$$




$$
\begin{array}{ll}
W_{i}^{\prime} \circ W_{j}^{\prime}=W_{k} & \rightarrow \text { symmetry operation, } \\
W_{i} \circ W_{j}^{\prime}=W_{k}^{\prime} & \rightarrow \text { antisymmetry operation. }
\end{array}
$$

If the motifs of the pattern are simultaneously black and white the so-called grey group is given (the antiidentity is element of the group). It should be mentioned that in antispace groups the antitranslation operation $T^{\prime}=T \circ 1^{\prime}$ has to be taken into consideration.

\section{Geometrical approaches for the description of crystalline interfaces}

An interface is geometrically characterized by the macroscopic and microscopic geometrical degrees of freedom (DOF). The macroscopic geometrical DOF describe the orientation relationships between the two crystal parts (3 variables) and the inclination of the interfacial plane determined by the interface normal (2 variables). An additional macroscopic DOF is necessary for describing bicrystals consisting of enantiomorphic crystals. The additional variable then characterizes the left or right handedness of the crystal.

The microscopic geometrical DOF describe the rigid body translation of the two crystal parts ( 3 variables). A fourth DOF describing the location of the average interface plane is necessary if either atomic basis is not monatomic.

Useful geometrical models for treating crystalline interfaces, especially homophase boundaries, are based on the concepts of

- the coincidence site lattice (CSL),

- the displacement shift complete (DSC) lattice,

- the O-lattice $[13,14]$.

Before describing the relations between the CSL, DSC, and O-lattices the derivative lattices, i.e. sublattices and superlattices will be defined. Consider two lattices $\boldsymbol{L}$ and $\boldsymbol{L}^{\prime}$ with bases $\boldsymbol{A}$ and $\boldsymbol{A}^{\prime}$

$$
A=\left(\begin{array}{c}
a \\
b \\
c
\end{array}\right), \quad A^{\prime}=\left(\begin{array}{c}
a^{\prime} \\
b^{\prime} \\
c^{\prime}
\end{array}\right), \quad \boldsymbol{A}^{\prime}=\boldsymbol{P A} .
$$

The following relations hold:

a) superlattice $L^{\prime}: p_{i j}-$ integers, $\operatorname{det}(\boldsymbol{P})>1$.

All the nodes of the superlattice $L^{\prime}$ are nodes of the original lattice $L$, but not all nodes of the original lattice $L$ are nodes of the superlattice $L^{\prime}$.

b) sublattice $\boldsymbol{L}^{\prime}: \boldsymbol{P}=\boldsymbol{Q}^{-1}, q_{i j}$ - integers, $\operatorname{det}(\boldsymbol{Q})>1$.

All the nodes of the original lattice $L$ are also nodes of the sublattice $L^{\prime}$, but not all the nodes of the sublattice $L^{\prime}$ are nodes of the original lattice $L$.

c) composite lattice $L^{\prime}$ :

Some elements of $p_{i j}, q_{i j}$ are fractional (special case).

Let us assume that two interpenetrating lattices $L$ and $L^{\prime}$ form a coincidence site lattice, this means a superlattice which contains all the lattice points in common to $L$ and $L^{\prime}$. The CSL is the intersection of the two lattices. It is characterized by a number $\Sigma$ which is the volume ratio of the unit cells of the CSL to the crystal cell. For cubic lattices $\Sigma$ can be any odd number. The value of $\Sigma$ is the reciprocal density of coincidence lattice sites referred to as the original lattice. A low ratio of 
$\Sigma$ means a high number of coincidence sites, whereas $\Sigma=\infty$ means an entirely random orientation. Special methods were developed to determine CSL possible in cubic, tetragonal, hexagonal, and rhombohedral lattices [13-19]. It should only be mentioned here that for describing heterophase boundaries with no exact CSL existing the so-called constraint CSL is introduced. The degree of matching is then described by two $\Sigma$ values one for each lattice.

The displacement shift complete lattice is the coarsest lattice which contains both crystal lattices as superlattices. The DSC lattice is the sublattice with the largest volume of the primitive cell. It is the union of the two lattices. The DSC lattice is the lattice of pattern conserving displacement. A displacement by a DSC vector of one crystal lattice with respect to the second causes a pattern shift which is complete. The DSC lattice defines the Burgers vectors of secondary dislocations (perfect grain boundary dislocations). According to Grimmer's reciprocity theorem [20] the DSC lattice for the crystal lattices $L$ and $L^{\prime}$ is the reciprocal lattice of the CSL formed by the reciprocal lattices $L^{*}$ and $L^{\prime *}$. The volume of the DSC unit is therefore given by $1 / \Sigma$. The relationship between the CSL and the DSC lattice is illustrated in Fig. 1, where two primitive cubic lattices are rotated about the [001] axis at $36.9^{\circ}$ forming a $\Sigma=5$ boundary.
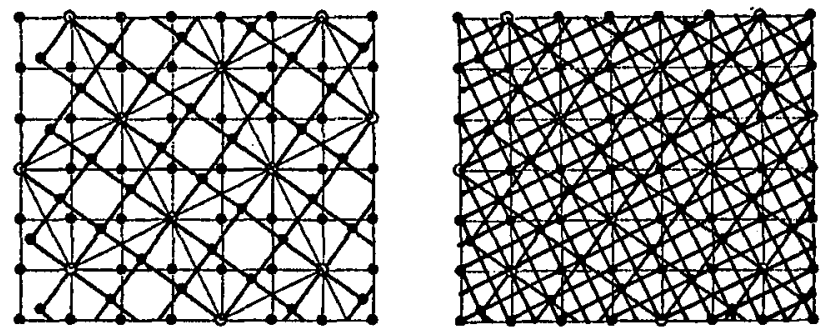

Fig. 1. CSL and DSC lattices for [001] $\Sigma=5$ boundary.

The O-lattice concept, as a generalization of the CSL, considers coincidences of points with the same internal cell coordinates in two interpenetrating lattices. Coincidences of points with the same internal coordinates are called O-points. Both crystal lattices are related by a linear transformation $x^{(2)}=\boldsymbol{A} x^{(1)}$. The O-elements are given as solutions to the basic equation $\left(I-A^{-1}\right) x^{(O)}=b^{(\mathrm{L})}$, where $b^{(\mathrm{L})}$ are the translation vectors of lattice 1 . The $\mathrm{O}$-lattice may be a point, line or plane lattice. Contrary to the CSL, the set of O-points continuously moves with a change of $\boldsymbol{A}$. The O-lattice concept was originally applied to describe the structure of low-angle boundaries and to study the interface crystallography (including interfacial dislocations). A general disadvantage of all geometrical approaches describing the interface structure is the restriction to the translational symmetry.

\section{Fundamentals of bicrystallography}

The symmetry of interfaces and of defects associated with the interface structure can be comprehensively described only if all symmetry properties possible of a crystal structure are applied. Using group theory various investigations of the 
symmetry of homo- and heterophase boundaries were carried out (see e.g. [21-25]). Useful constructions for the treatment of the symmetry of bicrystals. are

- the concept of dichromatic pattern (dcp) [21],

- the concept of dichromatic complex (dc) [24].

The bicrystal connection uses the theory of the symmetry of composites. In general, the symmetry of such a composite system is given by the intersection of the symmetry groups of the crystal parts forming the composite.

$G_{\mathrm{C}}=\left(\cap G_{\mathrm{BW}}\right) G^{\mathrm{C}}$.

Here, $G_{\mathrm{C}}$ is the space group of the bicrystal, $\cap G_{\mathrm{BW}}$ is the intersection of the space groups of the constituents (black and white crystal), and $G^{\mathrm{C}}$ is the symmetrizing group for homogeneous composites. As a consequence of the intersection the symmetry of $G_{\mathrm{C}}$ is reduced in comparison with the symmetry of the crystal parts (dissymmetrization). The determination of the bicrystal symmetry requires the knowledge of both the symmetrizing and dissymmetrizing operations.

The dichromatic pattern is the interpenetration of the lattices of the black and the white crystal, whereas the dichromatic complex is the interpenetration of the crystal structures. The dichromatic pattern can only be applied to determine the bicrystal symmetry of holosymmetric and symmorphic crystals. The dichromatic complex extends the symmetry analysis to non-holosymmetric and non-symmorphic crystals. An ideal bicrystal can be generated from the dichromatic constructions by selecting and fixing a section plane (interface plane), where white atoms are located on one side and black atoms on the other of the interface plane. The symmetry analysis has shown that heterophase boundaries can only exhibit ordinary space group symmetry, whereas homophase boundaries can also have antisymmetry space groups. All the possible space groups of bicrystals were calculated and tabulated in Ref. [24]. The complete determination of the interface symmetry requires the determination of the different stages of dissymmetrization, i.e., the transition from the ideal interface to the relaxed minimum energy interface structure. Each stage of dissymmetrization implies the occurrence of variants. According to the following transition scheme, four types of variants can occur [24]

$$
G^{*}(B)
$$

$$
O \quad G^{*}(p) \underset{C}{\longrightarrow} G^{*}(c) \underset{M}{\longrightarrow} G^{*}(b) \underset{R}{\longrightarrow} G(b) .
$$

$G^{*}(W)$

$\left(G^{*}(p), G^{*}(c)\right.$ are the dcp and dc, respectively, i.e., the intersection groups of Bravais lattices $G^{*}(B)$ and $G^{*}(W)$ and crystal structures $G(B)$ and $G(W)$, respectively; $G^{*}(b)$ and $G(b)$ are the groups of the unrelaxed and relaxed interfaces.)

The orientational $(O)$, complex $(C)$, morphological $(M)$, and relaxational $(R)$ variants can be determined by group decomposition (coset decomposition). The number of the possible variants is then given by the index $i=\left[G_{j}: G_{j+1}\right]$, where $G_{j}$ and $G_{j+1}$ are neighbouring space groups according to the dissymmetrization scheme.

The bicrystal symmetry concept was extended to describe all the possible crystal defects and interface defects (e.g., interfacial dislocations, disclinations, 
dispirations) which can arise in an interface [25]. Furthermore, special interface algorithms and computer programmes were developed and applied to determine the interface symmetry in heteroepitaxial systems [26, 27]. The so-called crystallochemical method includes the following:

- determination of the epitaxial orientation conditions based on a symmetry dictated energy extrema [22],

- determination of formation of degenerate domain epilayer structure and crystallographic type of possible defects in periodic boundaries.

\section{Applications}

It will be shown how the approaches of interface crystallography can be used to analyse interface structures experimentally observed.

The atomic structure of nanocrystalline particles formed by vapour deposition and subsequent annealing of amorphous thin films of germanium was studied by high resolution electron microscopy (HREM) [28, 29]. The HREM images of the nanocrystalline particles revealed the presence of a variety of coherent multiple twins on (111) planes. Preferential texturing with $\langle 110\rangle$ oriented crystallites prevailing was evidenced. These orientations allow one a revealing of the twin interface structure of the nanocrystals. The most striking features are circularly arranged microtwins which meet in three- or fivefold junctions parallel to the growth direction of the film. In some particles rather complex structures were observed, consisting of several fivefold twins with segments of subgrain boundaries in between. Different types of core structures of the intersecting regions are detectable in the HREM images. The HREM micrograph of an isolated multiple twinned particle with a fivefold twin junction is shown in Fig. 2.

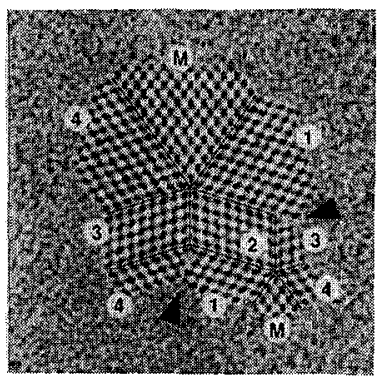

Fig. 2. High-resolution image (taken at $400 \mathrm{kV}$ ) of a nanocrystalline (110)-oriented multiply-twinned particle of germanium.

In the area of one twin two couples of stacking faults (marked by arrows) are present which are tetrahedrally arranged. A starting point of the analysis of multiple twins is the geometry of an ideal twin quintuplet. The twin quintuplet consists of five twins with a common [110] rotation axis. A successive rotation by $70.53^{\circ}$ around the [110] axis brings one twin component into parallel orientation with the other. This procedure of generating coherent twin boundaries leads to a circularly arranged structure with a lack of space filling. The gap of $7.5^{\circ}$ can be 
closed, for instance, by crystal defects such as incoherent twin boundaries, stacking faults, partial dislocations, etc.

The twin boundaries possible in the diamond structure can be classified according to the coincidence site lattice theory. The coherent primary twin (twinning occurs with $\{111\}$ as a twin plane) is a $\Sigma=3$ boundary. The role of multiple twinning and the specific properties of $\Sigma=3^{n}$ boundaries in crystals with fcc and diamond structures were extensively studied. Special computer simulation methods and programmes were developed to determine the crystallographic parameters of $\Sigma=3^{n}$ boundaries as well as their topological features [30]. The method used allows one to analyze the crystallography of triple and multiple junctions of the $n$-grains. A special group theoretical consideration was developed to describe the symmetry properties of triple junctions of boundaries [31]. In general, the group theory can be used for characterizing and numbering the interface operations which leave the given CSL invariant [32].

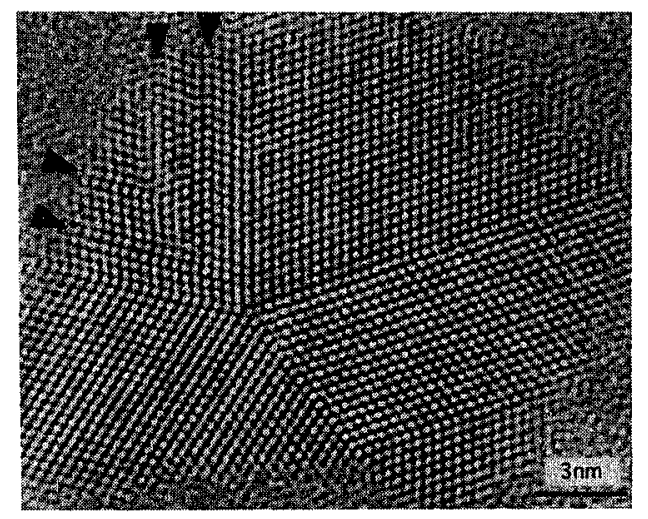

Fig. 3. Computer simulated model of twin quintuplets of germanium in (110) orientation.

The possibility of forming various types of multiple twins within a fivefold junction type is demonstrated in Fig. 3, showing a computer simulated fivefold multiple twin consisting of five tetrahedra. The twin boundaries within the ideal pseudo-pentagonal particle are four $\Sigma=3$ boundaries with a tilt angle of $70.53^{\circ}$, and one $\Sigma=81$ boundary (not shown in Fig. 3) with a tilt angle of $77.88^{\circ}$. The $\Sigma=81$ boundary is formed when there is a mismatch of $7.35^{\circ}$ between the $\{111\}$ planes. In the computer model the gap was closed by elastic deformation of the twins. The single tetrahedra were enumerated in the model, with $M$ signifying the matrix and numbers 1 to 4 denoting the order of twins. The adjacent components (as, e.g. matrix/first-order twin, second-/third-order twin) are structurally related by a rotation of $70.53^{\circ}(\Sigma=3$ boundaries). The various possibilities of combining the matrix with twins of different order lead to superposition structures with coincidence site lattices of types $\Sigma=3, \Sigma=9, \Sigma=27$, and $\Sigma=81$. These interface types will be formed according to the rule $\Sigma=3^{n}$ with $n$ as the order of the twins. The symmetry conditions of forming a CSL and the related symmetry 
TABLE

Crystallography of $\Sigma=3^{n}$ boundaries.

\begin{tabular}{c|c|c|c|c}
\hline \hline$\Sigma$ & $\begin{array}{c}\text { Cubic equivalent } \\
\langle 110\rangle \text { rotations } \\
\text { for } \Sigma=3^{n} \\
\text { boundaries }\end{array}$ & $\begin{array}{c}\text { Adjacent } \\
\text { regions in the } \\
\text { quintuplet } \\
\text { models }\end{array}$ & $H$ & $\Phi(\Sigma)$ \\
\hline 3 & $70.53^{\circ}, 109.47^{\circ}$ & $\mathrm{M}-1,1-2,2-3,3-4$ & $\overline{3} m$ & $6^{\prime} / m^{\prime} m m^{\prime}$ \\
9 & $38.94^{\circ}, 141.06^{\circ}$ & $\mathrm{M}-2,1-3,2-4$ & $2 / m$ & $m m^{\prime} m^{\prime}$ \\
27 & $31.59^{\circ}, 148.42^{\circ}$ & $\mathrm{M}-3,1-4$ & $2 / m$ & $m m^{\prime} m^{\prime}$ \\
81 & $77.28^{\circ}$ & $\mathrm{M}-4$ & $\overline{1}$ & $2^{\prime} / m^{\prime}$
\end{tabular}

of $\Sigma=3^{n}$ twin boundaries are given in Table. In general, the condition of forming a CSL is given by [31]

$$
\Phi(\Sigma)=H+R H, \quad R \not \subset \Phi_{1}, \quad H \subset \Phi_{1} .
$$

Here, $\Phi(\Sigma)$ is the symmetry group of the boundary, $H$ is the sublattice of the matrix phase $\Phi_{1}$ and $R$ is the CSL $180^{\circ}$ operator describing the boundary.

Image simulations of superimposed (11i) twins in [110] oriented germanium yielding to $\Sigma=3^{n}$ boundaries were carried out using the multi-slice programme of CERIUS HRTEM package [33]. Starting point of the contrast calculations was the geometry of the CSL models of $\Sigma=3^{n}$ twin boundaries. Under the conditions chosen the optimum defocus value (Scherzer focus) was $\Delta f=-405 \AA$. Figure 4 shows the superposition twin structures of $\Sigma=3, \Sigma=9, \Sigma=27$, and $\Sigma=81$ interfaces and the corresponding simulated structure images. The analysis of HREM images using the image matching technique has shown that such superposition structures exist in nc-Ge [33].

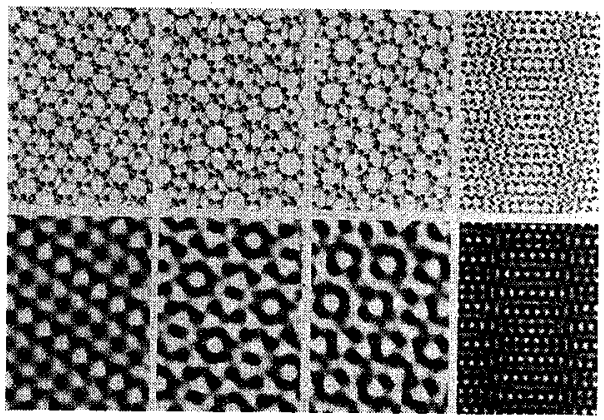

Fig. 4. Superimposed twin structures ( $\Sigma=3, \Sigma=9, \Sigma=27$, and $\Sigma=81$ interfaces) in (110) germanium and the corresponding simulated structure images for the Scherzer defocus. (Experimental parameters: accelerating voltage $400 \mathrm{kV}$; spherical aberration coefficient $C_{s}=1 \mathrm{~mm}$; focus spread $90 \AA$; beam divergence $0.65 \mathrm{mrad}$; objective lens aperture $0.50 \AA^{-1}$ ). 


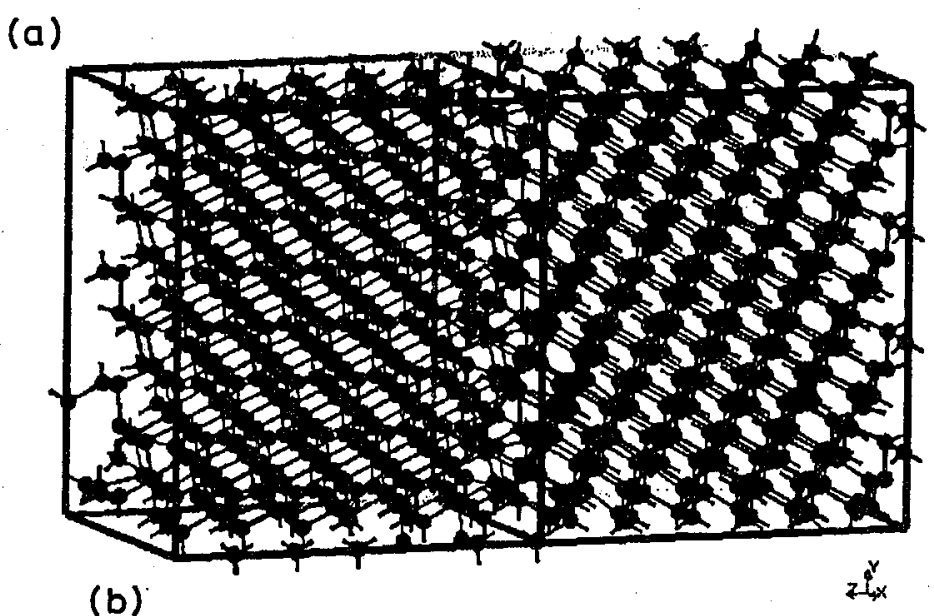

(b)

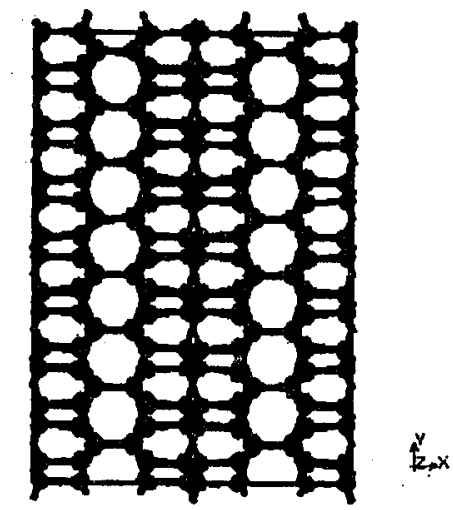

Fig. 5. Relaxed structure model of a $\Sigma=3$ interface in germanium. (a) Supercell containing 1152 atoms (interface plane marked). (b) Calculated atomic structure of a $\Sigma=3$ superimposed twin ((001). projection of the supercell).

The rigid CSL models did not consider the stress/strain state of the superimposed twinned part. In order to overcome these disadvantages molecular dynamics calculations have been started to determine the relaxed interface structure [34]. The relaxed structure model of a $\Sigma=3$ interface in germanium is shown in Fig. 5 . The supercell chosen contains 1152 atoms of germanium. The superposition of the adjacent twin parts is oriented in such a way that the (001) orientation of the supercell (Fig. 5a) is parallel to the (110) plane of the initially twinned germanium particles shown in Fig. 2. The atomic arrangement of the relaxed $\Sigma=3$ boundary (Fig. 5b), i.e. the structure of the interface plane energy minimized, obviously shows the generation of "linear channels" similarly to the unrelaxed superimposed structure. However, the atomic arrangement between the channels clearly exhibits deviations from the unrelaxed CSL model. The defocus series of the unrelaxed as well as relaxed structures of the $\Sigma=3$ boundary given in Fig. 6 clearly reveal the linear contrast features of the channels in the structure over the whole defo- 

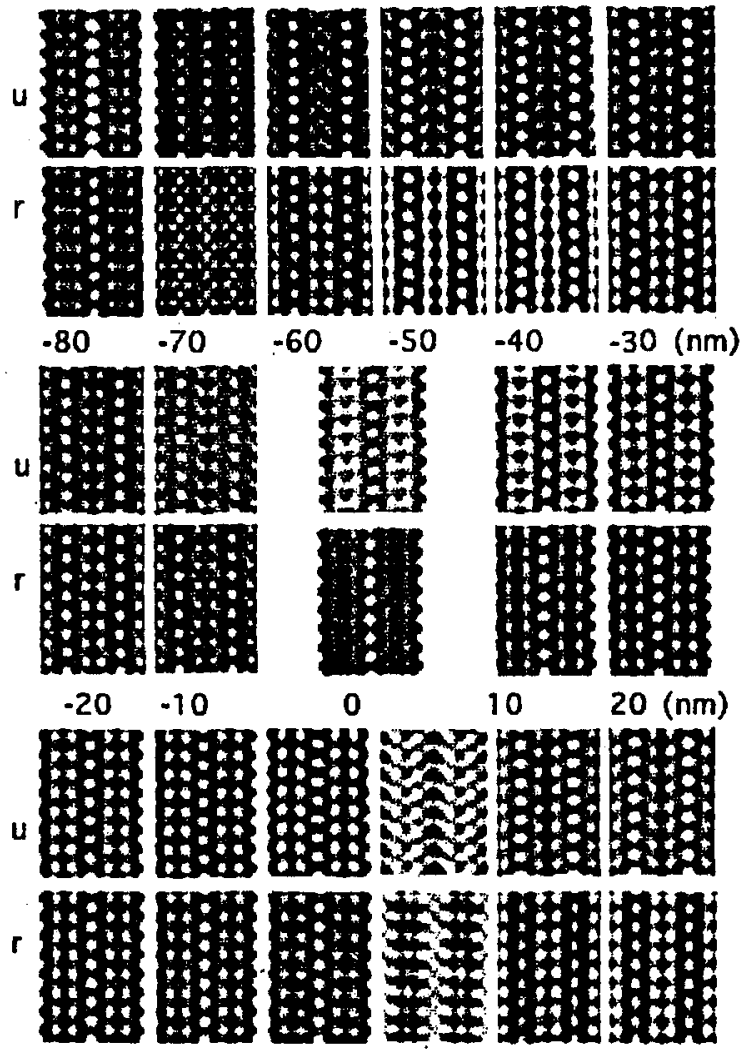

0

10

$20(\mathrm{~nm})$

$30 \quad 40$

50

60

70

$80(\mathrm{~nm})$

Fig. 6. Calculated defocus series ( $\Delta f$ in $\mathrm{nm}$ ) of HREM images of a superimposed twin structure of germanium in (110) orientation with a $\Sigma=3$ interface $(u-$ unrelaxed structure, $r$ - relaxed structure).

cus range. Comparing the simulated HREM images of the relaxed and unrelaxed structures reveals differences in both geometry and contrast of the basic pattern of the images.

\section{References}

[1] A.P. Sutton, R.W. Balluff, Interfaces in Crystalline Materials, Clarendon Press, Oxford 1995.

[2] Materials Interfaces, Eds. D. Wolf, S. Yip, Chapman\&Hall, London 1992.

[3] International Tables for Crystallography, Vol. A, Space Group Symmetry, 2nd ed., Ed. Th. Hahn, Kluwer Acad. Publ., Dordrecht 1989.

[4] Fundamentals of Crystallography, Ed. I. Giacovazzo, IUCr Texts on Crystallography, Oxford University Press, Oxford 1992.

[5] W. Steurer, in: Materials Science and Technology, Eds. R.W. Cahn, P. Haasen, E.J. Kramer, Vol. 1, Structure of Solids, Ed. V. Gerold, VCH, Weinheim 1993, p. 3.

[6] H. Burzlaff, H. Zimmermann, Kristallsymmetrie, Kristallstruktur, Verl. Rudolf Merkel Univ.-Buchhandl., Erlangen 1993. 
[7] B.K. Vainshthein, Modern Crystallography, Vol. 1, Symmetry of Crystals, Methods of Sructural Crystallography, Springer Verlag, Berlin 1981.

[8] C. Hermann, Z. Kristallogr. 69, 533 (1929).

[9] G. van Tendeloo, S. Amelinckx, Acta Crystallogr. A 30, 431 (1974).

[10] V. Janovec, Ferroelectrics 12, 43 (1976).

[11] A.V. Shubnikov, V.A. Koptsik, Symmetry in Science and Art, Plenum Press, New York 1974.

[12] V.A. Koptsik, Shubnikovskije gruppie, Izd. Moskovskogo Universiteta, Moskva 1966.

[13] W. Bollmann, Crystal Defects and Crystalline Interfaces, Springer Verl., Berlin 1970.

[14] W. Bollmann, Crystal Lattices, Interfaces, Matrices, Geneva 1982.

[15] H. Grimmer, W. Bollmann, D.W. Warrington, Acta Crystallogr. A 30, 197 (1974).

[16] G. Bleris, G. Nouet, S. Hagège, P. Delavignette, Acta Crystallogr. A 38, 550 (1982).

[17] H. Grimmer, Acta Crystallogr. A 45, 320 (1989).

[18] H. Grimmer, R. Bonnet, Acta Crystallogr. A 46, 510 (1990).

[19] H. Grimmer, Acta Crystallogr. A 45, 505 (1989).

[20] H. Grimmer, Scr. Metall. 8, 1221 (1974).

[21] R.C. Pond, W. Bollmann, Philos. Trans. R. Soc. 292, 449 (1979).

[22] D. Gratias, R. Portier, J. Phys. (France) 43, C6 (1982).

[23] G. Kalonji, J.W. Cahn, J. Phys. (France) 43, C6 (1982).

[24] R.C. Pond, D.S. Vlachavas, Proc. R. Soc. Lond. A 386, 95 (1983).

[25] R.C. Pond, in: Dislocations in Solids, Ed. F.R.N. Nabarro, Vol. 8, Elsevier, Amsterdam 1989, p. 1.

[26] A.V. Andreeva, B.L. Meiler, Cryst. Properties Preparation 36-38, 358 (1991).

[27] A.V. Andreeva, Mater. Sci. Forum 69, 111 (1991).

[28] H. Hofmeister, T. Junghanns, Mater. Sci. Forum 113-115, 631 (1993).

[29] H. Hofmeister, T. Junghanns, in: Proc. High-Resolution Electron Microscopy Fundamentals and Applications - Autumn School 1991 of the Int. Centre of Electron Microscopy, Eds. J. Heydenreich, W. Neumann, Elbe Druckerei, Wittenberg 1992, p. 245.

[30] A.V. Andreeva, A.A. Firsova, Internal report of IMT, Russian Academy of Sciences, Chernogolovka.

[31] G.L. Bleris, Th. Karakostas, Acta Crystallogr. A 45, 297 (1989).

[32] D. Gratias, R. Portier, M. Fayard, Acta Crystallogr. A 35, 885 (1979).

[33] W. Neumann, H. Hofmeister, J. Heydenreich, Phys. Status Solidi A 146, 437 (1994).

[34] W. Neumann, H. Hofmeister, D. Conrad, K. Scheerschmidt, S. Ruvimov, Z. Kristallogr. Suppl. 9, 308 (1995). 